\title{
CONTRA LA DESPOLITIZACIÓN DE LA MEMORIA. ENTREVISTA CON CARLOS ANTONIO AGUIRRE ROJAS, A PROPÓSITO DE LOS 40 AÑOS DE MAYO DEL 68
}

\author{
John Jaime Correa Ramírez ${ }^{1}$ \\ Universidad Tecnologica de Pereira. RUDECOLOMBIA \\ jjcorrea@utp.edu.co
}

Recepción: 30/05/2008

Evaluación: 1/06-15/09/2008

Aprobado: 29/09/2008

Artículo de Reflexión

\section{RESUMEN}

Con motivo de la celebración de los 40 años de mayo del 68, se realizaron algunas conferencias con invitados internacionales en la Universidad Tecnológica de Pereira. Se aprovecho la visita del historiador mexicano Carlos Antonio Aguirre Rojas, para realizar una entrevista en la que ampliara sus conceptos respecto al significado histórico de Mayo del 68 , su pervivencia en los imaginarios de muchos movimientos estudiantiles del presente, y al mismo tiempo, sus diferentes percepciones respecto a la historiografía tradicional y al movimiento zapatista.

Palabras Claves: Mayo del 68, memoria, resistencia, historiografía tradicional, contra-historias, nuevos movimientos sociales.

${ }^{1}$ Historiador. Magister en Ciencia Política. Profesor Universidad Tecnológica de Pereira (UTP). Estudiante XIV Promoción del Doctorado en Ciencias de la Educación, Rudecolombia - CADE Pereira. Miembro del grupo de investigación Políticas, Sociabilidades y Representaciones Histórico - Educativas (PSORHE), Categoría A Colciencias. 


\title{
AGAINST MEMORY DESPOLITIZATION. INTERVIEW WITH CARLOS ANTONIO ROJAS AGUIRRE: 40 YEARS AFTER MAY 68
}

\author{
John Jaime Correa Ramírez \\ Universidad Tecnológica de Pereira. RUDECOLOMBIA \\ jjcorrea@utp.edu.co
}

\begin{abstract}
On occasion of the 40 years after May 68 celebration, some lectures were given by international guests at Universidad Tecnológica de Pereira. Mexican historian Carlos Antonio Aguirre Rojas conducted an interview in which he extended his concepts regarding the historical significance of May 68 , the persistence in the imagination of many student movements for the time being; at the same time, their different perceptions regarding the traditional historiography and the Zapatista movement in Mexico.
\end{abstract}

Key Words: May 68, memory, endurance, traditional historiography, antistories, new social movements.

\section{INTRODUCCIÓN}

\section{Una corta referencia académica de Carlos Antonio Aguirre Rojas}

Mexicano, con postdoctorado en la Escuela de Altos Estudios en Ciencias Sociales de París, Carlos Antonio Aguirre Rojas es sin duda uno de los historiadores "más combativos" -siguiendo la idea de Lucien Febvre- de las dos últimas décadas en América Latina. Ha publicado, entre otras obras: América Latina: Historia y Presente; Los Annalesy la bistoriografía francesa; Fernand Braudely las Ciencias Humanas; Antimanual del mal historiador o ¿cómo hacer hoy una buena historia crítica?; Mandar obedeciendo; Chiapas Planeta Tierra. Es, así mismo, el director de la revista "Contrahistorias: la otra mirada de Clío".

Crítico acérrimo de la historiografía oficial en su país, Aguirre Rojas, propone llevar a cabo una doble tarea "transformadora" en el campo de los estudios históricos: "en primer lugar, un trabajo sistemático de crítica permanente de esa historia oficial, positivista y tradicional, trabajo que al mismo tiempo que denuncia y 
demuestra las inconsistencias de y la pobreza de los resultados historiográficos producidos por esta historia limitada que ha sido dominante... hasta el día de hoy, hace evidente también la clara función conservadora del statu quo que ha cumplido y que cumple este mismo tipo de historia perezosa y complaciente con los actuales grupos y clases dominantes. Pero también y en segundo lugar, es ahora necesario mostrar con claridad los nuevos rumbos por los que debe transitar la nueva historia que urge comenzar a elaborar, explicando con paciencia y detalle el conjunto de herramientas intelectuales y de puntos de de apoyo que habrá que utilizar en la construcción de esa otra historia diferente y crítica..."2.

Su énfasis, que podría denominarse "historia-denuncia", propugna por un historiador "de pié ante el presente". De ahí la importancia de conocer sus reflexiones sobre las memorias de Mayo del 68 -que él denomina "lecciones de una posible historia contra-radical”- y sobre la situación política en América Latina; sus aportes son muy significativos para entender el rumbo de las políticas neoliberales y las alternativas que desde los movimientos sociales tradicionales y emergentes se pueden ir consolidando como reductos de esperanza por una educación más incluyente y democrática en nuestro continente.

JC: Carlos, ante todo, muchas gracias por aceptar esta entrevista con motivo de los 40 años de Mayo del 68. Escuchando tu charla de ayer en el auditorio Jorge Roa Martinez de la Universidad Tecnológica de Pereira, tú hablaste de que Mayo del 68 tenía que empezar a analizarse en términos de una periodización histórica de mediana duración y no como algo coyuntural o simplemente "acontecimental", en tanto seguia teniendo una profunda significación sobre muchos de los movimientos estudiantiles del presente. Yo tenía deseos de preguntarte: ¿se podría decir que Mayo del 68 "sigue sucediendo" o si como proceso histórico es algo que se inició, pero aún no se ha cerrado?

CA: Es muy interesante esa idea, aunque es probable que también pueda tener muchos detractores, especialmente entre los críticos desencantados de Mayo del 68. Lo cierto es que si hemos venido a conmemorar estos 40 años no es sólo por algo simbólico, sino porque los acontecimientos que desencadenaron toda esta serie de expresiones novedosas entre el estudiantado del mundo entero siguen aún resignificándose continuamente. Incluso lo primero que habría que señalar es que quizás Mayo del 68 se adelantó a la globalización. Al menos se puede decir que fue algo que tuvo dimensiones "globales" y "complejas", para utilizar términos muy de moda en nuestra jerga académica.

Por ejemplo, habría que señalar-quizás de un modo paradójico- que mayo

${ }^{2}$ AGUIRRE, Rojas. (2002): Antimanual del mal historiador o ¿cómo hacer hoy una buena historia crítica?, Bogotá: Ediciones desde abajo, pp. 14-15. 
no es acontecimiento en un sentido literal, que incluso éste se inicia en China en 1966 - con la Revolución Cultural- y va a durar otros 10 años hasta 1976. En Argentina va a ser en 1969, con el “Cordobazo" Argentino o en Italia también va a comenzar en 1969 y se va a prolongar hasta los años 70's. Este acontecimiento concebido así en términos simbólicos muy amplios es un acontecimiento que tiene que ser evaluado por los historiadores y para esto Fernand Braudel nos dice que hay acontecimientos que podríamos llamar efímeros, acontecimientos históricos inmediatos, que se encienden y se apagan en una temporalidad muy corta, que duran sólo unos días, unas semanas, incluso unos meses, pero después se acaban. Yo creo que Mayo de 1968 es un "acontecimiento-ruptura”, como suelen decir los franceses, que marcó no solamente a la generación que lo vivió, no solamente a los muchachos que tenían entonces 15, 20, 25 o 30 años y que siguen hoy impregnados de su espíritu contestario y de rebeldía, sino que ha sido un acontecimiento tan importante, tan fuerte, que empezó a marcar a todas las generaciones que han venido después durante estos últimos 40 años que han transcurrido desde entonces. Yo creo que es un acontecimiento histórico que tiene una densidad que se inscribe, para hablar un poco en términos todavía de Braudel, más allá de lo coyuntural, aunque no sabemos si tendrá un impacto de larga duración, porque como bien se sabe, los fenómenos de larga duración son de un impacto o permanencia de más de 100 años, por lo menos. Pero es indudable que Mayo del 68 es un acontecimiento que abre una nueva coyuntura cultural en la que se despliegan toda una serie de efectos fundamentales -me parece que esto sí es evidente-, y que además tuvo la capacidad de articularse con otro tipo de luchas políticas y contraculturales.

JC: ¿Qué opinas de los eventos que se está realizando en Colombia y a nivel mundial en conmemoración de Mayo del 68? ¿Qué opinas respecto a cierto desencanto que hemos podido percibir entre algunos ponentes? ¿El desencanto es más de tipo politico o es un desencanto cultural ante la pregonada crisis de las ideologías y la carencia de nuevas vanguardias, especialmente en un ámbito como el universitario que a diario lo vemos ceder ante las presiones neoliberales?

CA: Yo pienso efectivamente que esta postura no es inocente y es un poco triste ver líderes históricos, por ejemplo, como Daniel Cohn-Bendit, que jugó un papel importante en aquellos años y que fue llamado "Dany El Rojo" en aquellas agitadas épocas, quien incluso tuvo que huir de Francia, que llego hacer acusado de ser judío, por lo cual llegó a realizarse una manifestación en París donde todos los estudiantes portaban pancartas que decían "Yo también soy judío”, o sea, como puedes ver, en torno a su figura y su rol político se dio un proceso de identificación muy grande. Y es muy triste ver que ahora que se cumplen 40 años, él publique un libro en el que se pretende olvidar Mayo del 68 y que afirme la tesis de que todo aquello por lo que lo que los estudiantes luchaban ya ha sido logrado y que por tanto no es necesario insistir en el 68.

Yo creo que esta visión es una visión muy miope y empobrecedora de lo 
que fue el 68 porque ¿qué es lo que estaba planteando por ejemplo mayo del 68 ? Entre muchas otras cosas otras cosas yo creo que en el centro lo que impugnaba era el modo en que se reproducía la cultura dentro de las sociedades contemporáneas actuales. Ese yo creo que es el centro del valor de mayo del 1968: haber puesto en crisis, haber puesto en cuestión a las tres instituciones fundamentales de ese momento: en primer lugar a lo que es la estructura de la familia, que es la primera formadora de valores, la primera formadora de ciertos códigos culturales, la que crea el primer saber o los primeros conocimientos de la gente. En segundo lugar, a la escuela naturalmente, a los mecanismos tradicionales de la transmisión de los saberes y también de desarrollo y reproducción de la ideología dominante. Y en tercer lugar lo que entonces era una realidad incipiente 40 años atrás, pero que hoy han adquirido una dimensión fundamental dentro de nuestras sociedades, que son los medios de comunicación. Yo creo que si vemos a estas tres instituciones, que son los tres espacios fundamentales donde se produce y se reproduce la cultura contemporánea, los 3 tienen un antes y un después marcado por el 68. Yo creo, por ejemplo, que la familia patriarcal, autoritaria, machista antes de 1968 era un estructura poco cuestionada, pero en los últimos 40 años es una institución absolutamente en crisis. Se podrían ofrecer datos interesantes para corroborar lo anterior. En México, por ejemplo, la tasa de divorcios que existía antes de 1968 era mínima, era menos del 1\% de las parejas; ahora hay una estadística que dice que de cada 10 parejas que se constituyen en México solamente 3 sobreviven al primer año de matrimonio, o sea, de 10 parejas que se constituyen 7 se disuelven.

JC: Creo que también Mayo del 68 two muchas implicaciones en función de recrear el imaginario politico y, en particular, la misma construcción del poder.

CA: Claro - pero para concluir lo anterior- también yo creo que a nivel del poder autoritario, la escuela sufre una crisis total y los medios de comunicación inciden sobre el poder de igual manera, si se toman en cuenta algunos eventos como Vietnam o la muerte de Martin Luther King. Ahora bien, retomando tu inquietud es válido decir que en la medida en que Mayo de 1968 es sobre todo una revolución cultural, transforma también la cultura política entonces dominante. Podemos citar las famosas frases de la "imaginación al poder" o incluso los aportes de un Michel Foucault desde sus teorías de la microfísica del poder que arremetían tanto en dirección de las burocracias capitalistas como las del socialismo soviético-. Pero así mismo creo que no es una casualidad que después de 1968 se inventó un término que antes no existía y que incluso había sido impensable para la actividad política previa al 68, que es el concepto de clase política: la idea de decir "todos los políticos juntos forman una sola unidad" era impensable antes del 68, porque se pensaba que los políticos de izquierda eran muy distintos de los políticos de derecha; después del 68 se impone poco a poco la idea de que tal vez todos los políticos comparten ciertos intereses, sin 
importar si son de izquierda, de derecha o de centro, y en ese sentido responden a ciertas prácticas y a ciertos códigos. Yo creo entonces que la política también entró en crisis, al evidenciarse que el político utiliza a la gente para conseguir su voto y una vez que se encumbra se olvida de ella, o esa idea de que el político tiene reuniones secretas de alto nivel donde deciden la suerte de los movimientos y la suerte de los problemas políticos con unos intereses que no se corresponden con el de la voluntad general, se hizo también muy fuerte.

Ahí se presenta entonces un cambio muy fuerte de la cultura política, en el sentido de cuestionar a la política como algo separado de lo propio social y cuestionar a la política como algo que si no está vinculada con la ética y con ciertos principios no tiene sentido. También en el plano de la cultura política contemporánea, Mayo del 68 es una revolución profunda.

JC: Ayer cuando planteabas -en una mirada más en prospectiva- todos esos legados del 68 en América Latina, me asombró mucho -te cuento que quedé un poco confundido- esa mirada crítica tuya no sólo frente a México o Colombia, paises que tú dices que "son las grandes vergüenzas a nivel latinoamericano", sino incluso frente a procesos como Venezuela, Bolivia, Ecuador, a quienes señalabas de un "socialismo socialdemócrata tibio". ¿En tu perspectiva de historiadory también de hombrepolitico, ves más opción en la revolución o en más estado social?

CA: Es muy oportuna tu pregunta y aprovecho para explicar el sentido de mi intervención ayer. Yo sin duda creo que naturalmente América Latina está poco a poco y lentamente mutando hacia la izquierda, de eso no me cabe duda. Pero yo creo que aquí la pregunta que debemos hacernos es ¿cuál es el factor principal que está haciendo que en América Latina después de haber tenido en los años 70's y 80's dictaduras militares tan sangrientas o regímenes de partido único -como el PRI en México-, habiendo tenido gobiernos muy conservadores, al igual que la dictadura de Pinochet, etc., de pronto, en los últimos 10 o 15 años hemos pasado a tener gobiernos muchísimo más preocupados por los problemas sociales? Yo creo que la causa fundamental que explica esto es la creciente fuerza que están empezando a tener en toda América Latina los movimientos populares. Aclaro que en este sentido fue que afirme que Colombia y México son la vergüenza; además mediante procesos que en cada caso había que explicar de manera comparada. Yo sí creo, por ejemplo, en México a raíz de un fraude electoral verdaderamente monumental se impuso un gobierno de derecha y ultraderecha. $Y$ es por eso que nuestro gobierno está haciendo las cosas mal, que no está respondiendo a las expectativas populares. Pero no sólo eso, que está hundiendo a México a una situación más difícil en términos económicos. La economía mexicana se está volviendo cada vez menos viable. Al mismo tiempo este gobierno está incrementando, en vez de tratar de reconstruir el tejido social y de tratar de despolarizar la situación, la 
está acentuando. Los gobiernos de derecha siempre hacen eso y dicen después de mí el diluvio -el presidente colombiano habla de "hecatombes"-. La idea que te venden es un poco como nos quedamos al precio que sea.

En Colombia yo creo que esto si tiene que ver con otros factor. La situación larguísima en el conflicto de guerra civil casi encubierta de 40 ó 50 años, que ha atentado contra muchas formas de participación popular, incluidos los movimientos estudiantiles. Estos gobiernos de derecha no resuelven los problemas y por eso la gente está buscando otras alternativas, de ahí que estos movimientos sociales están cobrando fuerza. Sin embargo yo diría que gobiernos como los de Hugo Chávez, de Evo Morales o de Lula, traicionaron las expectativas que se habían depositado en ellos. De Rafael Correa habría que decir un tanto más. Lo cierto es que las clases populares y muchos de los nuevos movimientos sociales están buscando salida no neoliberales, salidas distintas. Yo diría creo que todos estos gobiernos son como una etapa de transición en América Latina, porque lo que ellos están haciendo es tratar efectivamente de ser un poco más atentos a los reclamos sociales, tratar de despolarizar la situación de sus países, tratar de atender un poco más el gasto social, pero yo creo que en una perspectiva demasiado limitada; por eso decía que eran tibios gobiernos socialdemócratas. Es decir lo que ellos hacen es, en esencia, mantener las mismas políticas neoliberales pero tratando de parcharlas con una mayor preocupación por el gasto social.

\section{JC: ¿Estás hablando de "neopopulismo"?}

CA: No propiamente. El terminó quizás no iría bien. Más bien yo diría que es una especie de neoliberalismo atenuado. Los gobiernos de derecha del neoliberalismo salvaje o sea crudo y despiadado, no les importa las consecuencias ni a corto ni mediano plazo. Mientras que estos ya son parte de una visión digamos- de una burguesía más nacionalista, más preocupada del mercado interno y que dicen: "no nos conviene seguir polarizando la situación porque esto va a estallar y se nos va a salir de las manos". Mantienen las políticas neoliberales pero atenuadamente, las parchan o las complementan con un poco de gasto social, es lo que es el programa hambre cero de Lula, por ejemplo, en Brasil. Eso era lo que proponían las políticas del ex - candidato presidencial Andrés Manuel López Obrador en México, de darle becas a las mujeres solteras, becas a los de la tercera edad, o sea, los pensionados que viven miserablemente en México y abrir más universidades, porque en México la universidad ha sido muy popular, pero ahora las clases populares no están teniendo acceso a la universidad y eso crea un problema social muy grande.

Por eso digo que son gobiernos tibios socialdemócratas y es más desde la fuerza de los movimientos populares que sigue aumentando donde se están buscando salidas muchísimo más radicales y volvemos a la última parte de tu 
pregunta. Yo creo que la solución no es más Estado -reformista o interventor-, no, por el contrario, la solución es más bien la construcción de un tipo de Estado radicalmente diferente al que existe, un estado que no sea capitalista y que debiera formarse sobre la base del principio de "mandar obedeciendo", algo que la humanidad no ha conocido hasta hoy o lo ha conocido en pequeña escala en los Caracoles Zapatistas o en los asentamientos de Los Sin Tierra. Pero yo creo que lo que tenemos que redefinir es los términos de la política. Debe haber un grupo o una clase política que es la que decide y la que "manda mandando" -estoy aquí usando los términos de los compañeros zapatistas- y la mayoría de nosotros los ciudadanos tenemos que obedecer porque supuestamente al elegirlos con nuestro voto les delegamos la capacidad de decidir sobre nuestros asuntos; o tendríamos que devolvernos a las ideas sobre la democracia griega original, que definían la democracia, demos: pueblo; kratos: gobierno, o sea un gobierno del pueblo que no piense en otra cosa más que en autogobierno popular. Entonces me pregunto si no deberíamos de volver más bien a la idea de que a nosotros nos corresponde decidir por nosotros mismos la suerte de nuestros destinos y esa es la idea del mandar obedeciendo, la idea de devolverles a los ciudadanos comunes y corrientes la capacidad de decisión y es a eso hacia donde tenemos que ir; no hacía más Estado ni a un Estado más Social, sino una forma radicalmente distinta de Estado que a lo mejor ya no se llama Estado, porque es tan distinta que será más bien basado en el principio de "mandar obedeciendo".

JC: ¿No se trata mas bien de una especie de nuevo contrato social o no lo quieres encasillar en este concepto por seguir siendo todavía muy occidental este concepto?

Claro. Se puede plantear inclusive en esos términos de teoría política clásica de Rousseau, porque además incluso los zapatistas lo dicen y es un tanto curioso, porque ellos se preguntan constantemente: ¿Por qué estamos luchando?, y ellos dicen: "En una primera fase inmediata estamos luchando por una nueva constitución". Pero justo a la hora en que se les ha pedido que aclaren que entienden por una nueva constitución, dicen: "Es un nuevo pacto social, tenemos que hacer un nuevo acuerdo entre todos los grupos que formamos una nación y decir cómo queremos autogobernarnos; y decir que relación queremos tener; y decir cómo queremos que se gestione, se produzca y, sobre todo, se distribuya la riqueza de esta país".

Ellos dicen una metáfora que a mí me gusta mucho: "No es justo que unos pocos concentren toda la riqueza y los otros trabajemos para ellos; tenemos que estar todos parejitos. Cada quien debe de vivir del fruto de su propio trabajo”. Entonces ahí están planteando obviamente lo que el socialismo reivindicaba, es decir, una socialización de los medios de producción etc. Pero ya no es al estilo clásico, ya que están planteando una redistribución de la riqueza que impida que sean unos poquiticos y escandalosamente ricos y la inmensa mayoría pobre. En 
este sentido si es un nuevo pacto social, que además involucra nuevamente la cuestión de la voluntad general o la voluntad popular bajo el designio de "mandar obedeciendo".

\section{JC: ¿Cuál es el lugar de ese esquema de "mandar obedeciendo" desde la academia?}

CA: Es una pregunta muy interesante. Yo creo que quien entendió muy bien este problema fue Michel Foucault. Él vive muy de cerca los acontecimientos del 68 y él dice que lo que nos demuestra el 68 es un poco la idea de la muerte del intelectual ilustrado que concentra todo el saber y efectivamente rompe esta idea de que el intelectual sabe y la sociedad tiene que escucharlo; hay que decir que es lo mismo que sucede a nivel de la escuela: todo el saber está depositado del lado del maestro y todo el colectivo de estudiantes no sabe nada, y mientras el maestro habla los estudiantes escuchan, el maestro afirma y los estudiantes tienen que aprender, o sea, es la vieja del magíster dixit, que como se ve, es un argumento de autoridad, ya que como lo dijo el maestro por lo tanto es verdad. Pero si en el seno de la institución académica se rompe esta idea y se pasa mas bien a la idea que dice: el colectivo estudiantil en general siempre sabe más que el maestro porque siempre 30 o 40 personas casi a priori saben más que un solo individuo -por preparado que él esté- y esto se difunde como tendencia general, entonces si damos un giro incuestionable, como en efecto vemos que el problema de toda la pedagogía contemporánea en los últimos 40 años se centra en cómo rescatamos este saber colectivo que está depositado y disperso en todo el colectivo estudiantil y lo actualizamos como palanca del conocimiento en generación del saber. Yo creo que esto cambia el rol del intelectual. A nivel de la sociedad sucede lo mismo: si se rompe esta idea del intelectual ilustrado sabelotodo, encarnación del saber que se pronuncia en las aulas o en los medios de comunicación, sobre todos los temas habidos y por haber, el intelectual entonces deja de ser como una especie de líder natural o de asesor o de personaje que marca las líneas directrices de un movimiento y se vuelve -como decía Foucault, por eso me gusta su planteamiento- en un simple acompañante de los movimientos.

Entonces, ¿cuál puede ser ahora la función del intelectual? El intelectual tiene que poner al servicio de las clases populares y de los movimientos sociales las herramientas de saber que él aprendió en la universidad, pero igualmente en una actitud mucho más humilde, que permita hacer emerger otras voces, otras posturas, en una actitud de decir: yo sé ciertas cosas por haber ido a la universidad que quizá la gente que no fue a la universidad no sabe, pero el saber de esta gente que no fue a la universidad es tan importante y tan fundamental como el mío. Creo que los retos actuales de la academia también pasan por enriquecer y complejizar las maneras de combinar ambos saberes para tratar de construir un saber nuevo, pero no soy yo el que viene a decir que es lo que hay que hacer 
y cómo hay que hacerlo. Creo entonces que esto es lo que se quiebra en el 68 y no es casual tampoco que la Revolución Cultural China tenía en el centro de su cuestionamiento esta división entre trabajo manual e intelectual.

JC: Interesante este planteamiento. Toda esta reflexión me hace recordar también esa reflexión que en un momento llegó a plantear un filósofo de la talla intelectual de Kant frente a la Revolución Francesa, en ese famoso texto ¿Qué es la ilustración? Michel Foucault planteaba que esta pregunta por la ilustración es una pregunta que la filosofía moderna no ba sido capaz de respondery de la cual tampoco ha podido desembarazarse. ¿ Las preguntas por Mayo del 68 sepueden encajar dentro de esa tendencia recurrente de interrogar el pasado desde elpresente de una modernidad todavía inconclusa o por una modernidad que siempre deviene en nuevos retos? ¿Sigue siendo pertinente preguntarnos de este modo? ¿Mayo del 68 nos liberó de una minoría de edad?

CA: Claro yo creo que sigue siendo pertinente, aunque también después de la Escuela de Frankfurt hay que asumir los límites de esa Ilustración. Pero hay que ir más despacio con todo esto. En el texto de Kant hallamos reflexiones que sin duda siguen siendo muy valiosas en relación con la forma como se pueden relacionar la voluntad y la autoridad desde un nuevo uso de la razón, que es tanto un hecho o un proceso en vías de desarrollo -es decir un ahora presente-, como una tarea o una obligación -que también nos insta desde el presente hacia el futuro inmediato-.

Pero la pregunta ahora ya no podría plantearse en los mismos términos. Después de que hemos vivido el inicio del ocaso de la razón ilustrada tenemos que entender que la "Nueva Ilustración" -si queremos hablar un poco de esa metáfora- tiene que ser necesariamente mucho más crítica y mucho más autocrítica, porque la razón ilustrada digamos que era crítica respecto de las formas de razón anteriores, pero era poco autocrítica consigo misma. Ahora, todo pensamiento social nuevo, toda ilustración nueva tiene que ser también profundamente autocritica, -yo insisto en esta cuestión- porque toda la Ilustración se basa en la idea de que hay una alta cultura y una baja cultura, y yo creo que esta idea también de una alta y baja cultura tiene que ser quebrada. El saber popular es el generador de la cultura de elite y aquí yo pensaría también en un historiador como Carlo Ginzburg, por ejemplo. Él ha redefinido muy bien cuál es la verdadera relación que hay entre cultura popular y cultura de elite, donde él nos muestra que no son dos universos separados, no son dos entidades que se generan cada una por su lado, son un círculo que interactúa todo el tiempo uno con otro, donde el elemento dominante no es como se afirmaba hasta hoy la elite, sino por el contrario el elemento dominante es la cultura popular, la cultura elite es simplemente una recodificación de la cultura popular, que se usa para legitimar el dominio de las clases dominantes. Entonces si entendemos las cosas así, tenemos que volver la mirada hacia este elemento 
nutricio generador de toda cultura que es la cultura popular. Yo creo que por ahí, bajo esta perspectiva, habría que replantear si se quiere usar tan literalmente esa metáfora de la Ilustración o si el papel de una nueva Ilustración tendría que ser no sólo crítica si no autocrítica.

JC: Ahora quisiera hablar brevemente de ese otro proyecto académico por el cual te he conocido y que valoro enormemente: el proyecto de la revista "Contrabistorias". La postura editorial que desde un comienzo ustedes han venido promoviendo me parece muy necesario para el desarrollo no solo de la historia sino de las ciencias sociales, pero al mismo tiempo, busca tener repercusiones en ciertos ámbitos sociales y políticos, es decir, los temas de la revista no se quedan en una simple discusión entre académicos, sino que también tiene que trasladarse a los grupos sociales, a los colectivos, a las comunidades, barrios, etc. ¿Esa idea de "Contrabistorias" cómo surgió?

CA: El término literalmente está sacado de Michel Foucault. En su libro "La genealogía del racismo", él habla un poco de la hegemonía de la historia oficial para contraponerle su idea de la genealogía. Él se refiere críticamente a la memoria oficial, que se construye vinculada a esta historia oficial del poder $-\mathrm{y}$ no siempre tan pública como pretende mostrarse y legitimarse- para oponerle el término de la "contramemoria". Recuerdo que Foucault sugiere en algún fragmento de su texto que la nueva historia que nosotros debemos hacer cuando trabajamos desde el punto de vista genealógico- es construir más bien "contramemorias" y entonces comienza hacer este juego que me parece fundamental, que es: frente a la historia oficial-que siempre nos muestra orígenes gloriosos- lo que la "contrahistoria" tiene que mostrar es las formas oscuras espurias y no siempre nobles, por ejemplo, de cómo se han construido las naciones. Se tiene que mostrar esa parte de la que nadie habla, que se encubre por múltiples intereses, porque rompe la tersura y la esencia del mito que envuelve la historia oficial. Las formas y contenidos de la memoria dominante siempre seleccionan y enaltacen a ciertos personajes o moldean los acontecimientos para que sean acordes a un desarrollo siempre progresivo, lineal, siempre ascendente y siempre maravilloso. Pero no hay una historia de Colombia o una historia de México que hable de los "lados malos", que hable de las masacres, que hable efectivamente de las guerras, que hable de cómo se obliga a ciertos sectores de la población o a ciertas regiones a integrarse a un proyecto nacional. Eso nunca lo habla nadie. Entonces, frente a esta memoria oficial hay que plantear una contra memoria.

De acá viene el término, aunque como anécdota, te cuento que en un primer momento pensamos en ponerle como subtítulo "Por una historia a contrapelo", es decir, el término de Walter Benjamín, que se emparenta con esta idea de la contrahistoria. Sin embargo nos decidimos finalmente por dejar como subtítulo "La otra mirada de Clío". Lo que queremos nosotros en 
"Contrahistorias" es dejar de ver esta historia en los términos gloriosos o tersos como nos la presentan la historia oficial, para pasar el cepillo de la historia a contrapelo y sacar esas historias marginales, ver a los grupos subalternos e inventariar constantemente aquellos temas históricos ausentes del debate académico, de los que nadie habla. En síntesis, la idea principal yo creo de Benjamín es ver no solamente la línea del pasado que resultó finalmente vencedor, sino las líneas de todos los pasado vencidos, que pudieron haber sido pero que no lograron imponerse, pero que por no haber logrado imponerse entonces desaparecen. Es más, hemos podido constatar que a pesar de que se trate de imponer una memoria a modo de historia oficial, persisten a través del tiempo muchas otras luchas reales, muchas otras luchas por la memoria, como sucede por ejemplo en los Balcanes y en tantas otras partes del conflictivo mundo del presente. Por eso nosotros quisimos desde el primer número que "Contrahistorias" ayudará a hacer emerger todos esos lados "malos" u olvidados de la historia de los que hablaba Hegel, ese lado negativo, esa parte -digamosde la historia crítica, esa ha sido un poco la tarea de nosotros en el proyecto de la revista, combinando aspectos teóricos con entrevistas y propuestas de nuevas miradas sobre conflictos y violencias a "contrapelo".

JC: Yo he asumido la invitación de "Contrahistorias" como la posibilidad de trabajar bistorias desde las márgenes, desde los invisibles, desde los que no están en las primeras planas de los periódicos, sino desde los que emergen opacamente desde las páginas rojas de la prensa, al final de las ediciones, en las crónicas rojas donde se denuncia a los anormales, lo anómico, lo que subvierte el orden. Siguiendo a Gabriel Restrepo, un conocido sociólogo colombiano, me he preocupado por identificar "los reversos de las urbanidades", de las historias civicas llenas de gestas de grandes prohombres, especialmente en Antioquia y más recientemente en el Eje Cafetero. Esto también me ha motivado a escribir mucho sobre historia barrial, historia de personajes más anónimos, incluso algunas historias en perspectiva de género. Sin ser un estudioso a fondo de las problemáticas que reivindica el feminismo, me he interesado por rescatar historias de mujeres de diferentes condiciones sociales para ponerlas en relación con contextos históricos más amplios, con historias de procesos solidarios o con otros procesos socio-culturales. Me gusta mucho este tipo de historias, que tiene que ver, sin duda, con la identidad o la biografía personal, y que también podriamos denominar "Bistoria desde abajo", pero be tenido siempre una inquietud: ¿"Contrahistorias" tiene implicaciones epistemológicas o sus implicaciones son más de tipo político, propias del tipo de "bistoria denuncia"? ¿O es un falso problema de dividir lo epistemológico y lo politico en este caso?

CA: Tiene pretensiones epistemológicas en el sentido que te decía de explorar la realidad o el pasado histórico a "contrapelo" para ver qué logra emerger, que logramos hacer visible. Y naturalmente tiene implicaciones políticas, como lo decimos desde el primer editorial de la revista. Yo creo que efectivamente nosotros queremos quebrar con esa idea falsa, que a mí me parece que es ridícula, pero que tenazmente se ha mantenido. México goza de 
un buen prestigio a nivel latinoamericano por sus escuelas históricas, pero en mi concepto, la Historia -con mayúscula- está supremamente atrasada en México. Se mantiene esa idea que la historia es el estudio de los problemas del pasado. Nosotros desde el primer editorial dijimos que si bien la historia es el estudio del pasado, también lo debe ser del más actual e inmediato presente. Esto implica reconocer que la historia es también lo inmediato, lo que estamos conversando ahora, lo que estamos viviendo, la compleja situación de Colombia hoy, de México, de Estados Unidos, la guerra en Irak, los conflictos que se están dando en Europa, el proyecto de la Unificación Europea, la crisis que hoy vive Rusia, las encrucijadas de China, de si se mantiene en el socialismo o si pasa a la economía de mercado, etc. Todo eso es historia viva que nosotros estamos viendo, que tiene muchas raíces en un pasado cercano o lejano y que por lo tanto debe ser analizada con las mismas herramientas del historiador y con la misma densidad con la que tanto intentamos analizar el pasado.

Entonces el abordar este objeto que podríamos llamar de la historia inmediata, sobre la historia todavía viva, nos lleva naturalmente al tema del compromiso político del historiador, que en los años 70 era una evidencia, la idea de que el científico social trabajaba en un contexto y tenía que responder a él y tenía que -de alguna manera- comprometerse y asumirse de manera crítica frente a sus propias afirmaciones. Esta era una evidencia que curiosamente se perdió en los años 80 y 90 bajo un mecanismo muy cuestionable, quizás un tanto posmodernista, de que el historiador tiene que ser neutral, para poder ser objetivo y cuando se dice objetivo se dice desapegado de los conflictos del presente. Esto en realidad no es posible, porque el historiador que pretende no tomar partido está tomando partido implícitamente por el lado o por el otro. Nosotros estamos en contra de eso. Al estudiar la historia inmediata con una perspectiva crítica y densa, necesariamente tenemos que asumir una posición clara sobre lo que está aconteciendo y decir: "la guerra de Irak la aprobamos o la condenamos y la aprobamos o la condenamos por tales y tales razones". Me imagino que ustedes en Colombia también deben vivir esta situación.

Nosotros decimos que en el caso de México la situación política y de violencia nos parece difícil y complicada en este sentido y por esta razón y estamos impulsando su transformación en tal vía, y eso es lo que ha hecho un poco la revista. Entonces si te das cuenta en los años más recientes hemos hecho números más políticos que logran analizar por ejemplo el "Movimiento de la Otra Campaña" de los Zapatistas, o tratando de ver lo que son los movimientos sociales en América Latina, tema al que le dedicamos el número 5. Total, la idea es abrirnos no sólo a los temas historiográficos sino a los temas del debate político visto con perspectiva histórica densa.

JC: En la perspectiva de la construcción del Estado Nación, que es uno de los temas 
"serios" de los historiadores -no sé si usted comparte opercibe esa sensación de que los historiadores nos ponemos muy recelosos o no aceptamos salirnos de ciertos moldes historiográficos cuando hablamos de la construcción del Estado Nación-, ¿"Contrabistoria" también tiene entonces su propia propuesta en esa lectura de la construcción de los estados naciones, sobre todo en América Latina?

CA: Claro. Pero es curioso que en América Latina lo empezamos a discutir justo cuando a nivel mundial comienza a entrar en crisis el Estado benefactor y el Estado Nación también. Yo creo que hay que tener en cuenta una cosa: la nación es una construcción burguesa, la nación es una construcción del capitalismo moderno y no había naciones de la Edad Media para atrás; había otras estructuras políticas, estructuras imperiales o reinos. Pero no hay propiamente la idea de nación. La idea de nación es una idea del capitalismo moderno y por qué digo esto, porque yo creo que, y esto es un poco la idea de Inmanuel Wallerstein, el capitalismo moderno está viviendo su crisis terminal y si está viviendo su crisis terminal colapsa también el proyecto de Estado Nación como proyecto civilizatorio. Yo creo que debemos empezar a movernos hacia un verdadero internacionalismo o un nuevo mapa político, con nuevas relaciones geopolíticas. El día de mañana ya no habrá naciones, como no las hubo hace 5 ó 6 siglos, como no las hubo incluso en América Latina hace apenas 2 ó 3 siglos.

Entonces el tema de Estado Nación es un problema histórico que debemos estudiar y que debemos investigar en sus consecuencias, en sus guerras, en sus exclusiones, en la cultura política autoritaria, en los dispositivos ideológicos que se manejan desde la escuela, etc. En "Contrahistorias" nos interesa seguirle la pista a esas evidencias de crisis, tanto desde abajo como desde arriba. Desde abajo por esta "re-emergencia" de identidades locales, de identidades de grupos, de identidades étnicas; qué cosa es lo que nos están diciendo este tipo de fenómenos que son del presente, pero que provienen de tiempo atrás; son identidades civilizatorias que fueron previas a la imposición de una cultura nacional, que nunca lograron ser asimiladas del todo a este esquema. Desde arriba diríamos que este mal llamado de la globalización - a mí el término no me gusta para nada-, pero digamos que este proceso de intensificación de los vínculos entre las economías, las comunicaciones, etc., demuestra que las estructuras intermedias son cada vez menos funcionales. Yo creo que debemos movernos hacia perspectivas mucho más profundas, en la que la idea de Estado Nación homogéneo e incuestionable no cabe.

JC: Y en esa misma medida, ese resurgimiento de lo local, de las identidades en plural, que muchos historiadores "serios" desdeñan porque afirman que lo local se pierde en su propia anécdota y que se pierde de vista el conjunto nacional-como si éste existiera o como si éste fuera empujado por un único mecanismo de manera simultánea-, me parece que en "Contrabistorias" tiene otra mirada, digamos más abierta.

CA: Claro, porque depende cómo lo veamos. Si es cierto que es un riesgo 
eso de lo anecdótico, aunque desde el punto de vista de la memoria oral es un filón interesante de explorar. Aquí yo pienso en un proyecto mexicano que quizá se conoce en Colombia y es el proyecto del historiador mexicano Luis González y González, el de la microhistoria mexicana. Este señor ha generado todo un culto entre viejos y nuevos historiadores, pero como te he dicho, nosotros somos muy críticos de la historia mexicana en general, y por eso, el tema del número 1 de "Contrahistorias" era sobre la microhistoria italiana porque la gente en México necesita conocer de otras formas de hacer microhistoria. Se puede hacer una historia muy chata, muy pobre, muy limitada, una historia puramente anecdótica de contar lo que está dicho, que en lugar de problematizar termina es siendo mitificadora y tranquilizadora, que incluso es como una especie de refugio en el pasado frente a las complejidades del presente. Esa historia si se queda en ese ámbito local, yo creo que no le interesa a nadie. Pero en cambio si seguimos la perspectiva de Marc Bloch, el historiador francés, que planteaba que las condiciones para que una historia cualesquiera, local o regional, sea una historia realmente científica, depende de las preguntas que se plantee esa historia. Él dice: "no es lo mismo plantearle preguntas locales a una historia local que plantearle preguntas universales a una historia local". Decía algo más o menos así: "si nosotros nos planteamos problemas de orden universal en el ámbito de una localidad hacemos una historia científica que le interesa a todos los historiadores del gremio, si nos planteamos preguntas del orden local para responderla con elementos locales eso le interesa a muy pocas personas, quizás a los habitantes de esa localidad y punto". Yo creo que "Contrahistorias" ve de esta manera el problema todas esas historias locales, que sin duda son de una riqueza extraordinaria. Los lenguajes vernáculos, por ejemplo, que ahora se pierden en el mundo por la homogenización lingǘstica, pero que ahora van desapareciendo poco a poco dentro de ciertos procesos de homogeneización cultural; a mí me parece que eso es un crimen, porque cada vez que un idioma se pierde se muere una conquista de la propia humanidad. Tenemos que defender todas esa identidades, pero no para quedarnos en lo local y olvidarnos de lo supra local, sino al contrario, podríamos crear un especie de lenguajes mixtos que integren todas las lenguas locales en un espacio de convergencia cultural, donde todas ellas puedan reconocerse y dialogar entre sí. Estoy siguiendo un poco el argumento de Benjamín, él se preguntaba si se podría construir una lengua universal y si se eso se podría hacer, bajo qué condiciones se podría asegurar espacios o semánticas de co-existencia, que es la base de la comunicación. Yo creo que esta es la pregunta que nosotros debemos plantearnos: ¿Cómo podemos crear una estructura donde toda esa riqueza local extraordinariamente diversa y grande no se pierda pero tampoco se subsuma en lo puramente local, si no que permita crear efectivamente un diálogo plural y universal? En esa perspectiva veríamos el problema nosotros en "Contrahistorias".

JC: Hay una pregunta que no puedo dejar de planteártela: ¿Cómo le apuesta 
"Contrahistorias" a los problemas de la interdisciplinariedad? Es que en ocasiones la interdisciplinariedad se vrelve un argumento-o incluso, unapose-sin un buen sustento metodológico, que muchas veces para lo único que sirve es para desacreditar el trabajo de muchos de los que seguimos trabajando creativamente con los métodos de nuestras disciplinas de formación, en este caso, con la Historia. En este sentido me atrevo a decir que la historia ha estado muy abierta a reconocer sus limitaciones teóricas, ha aprendido de sus limitaciones y mutaciones de método, y se ha abierto a la necesidad de entender los lenguajes, los métodos, los problemas de otras disciplinas, en un diálogo abierto que ya proponían autores como Lucien Febvre en "Combates por la Historia" y Fernand Braudel, en su texto clásico "La Historiay las Ciencias Sociales".

CA: Nosotros somos naturalmente muy críticos de las visiones disciplinarias, que a veces terminan siendo incluso dogmáticas frente a la idea de realidad, de cambio social, de fuentes, etc. Pero creo que eso se ha venido pregonando de tiempo atrás, digamos desde toda la historia crítica, es decir, desde Marx hasta hoy. Hemos criticado, unos más que otros, esa división que me parece paralizante y poco efectiva en términos intelectuales, en la que el economista cree que todos los hechos se explican a partir de lo económico; el sociólogo cree que todos los hechos son solo hechos sociales y desatiende lo cultural, lo económico, lo geográfico, etc.; el geógrafo cree que lo determinante es el medio ambiente y las bases naturales. Nosotros adoptamos una posición menos taxativa o nominalista. Es que hay muchas modas: historia ambiental, historia de la salud, etc., pero en el fondo todo es historia y lo que importa es la buena historia, bien investigada, bien contrastada, problematizadora y bien escrita, plena de sugerencias. Por eso cuando se menciona la inter, la multi, la pluri, la transdisciplinariedad, seguimos un poco la perspectiva de Fernand Braudel. Él hacía muchas bromas al respecto, comentaba, por ejemplo, que cuando venían sus colegas a hablarle de todos esos términos, él les refutaba diciéndoles que la idea que se le venía a la mente era algo así como la historia de matrimonios convenidos entre disciplinas, es decir, vamos a casar a la historia con la economía para que nazca la historia económica, vamos a juntar a la antropología con la sociología y que salga la antropología social. Entonces Braudel, que era muy radical y muy osado, decía: a mí la idea de matrimonios convenidos, muy formales, muy bonitos, muy bien establecidos, no me gusta para nada. Yo estoy por la promiscuidad generalizada. ¿Qué quiere decir esto? Él decía más bien que no se puede pensar la prioridad de los compartimentos estancos, a la manera de la ciencia de lo económico, la ciencia de lo psicológico, la ciencia de lo político, etc., si no más bien, una ciencia de problemas y los problemas son unidisciplinares, es decir, los problemas atraviesan una multiplicidad, una posibilidad de miradas, no con el ánimo de relativizar, sino de comprender de manera calidoscópica cualquier problema importante, el que tú quieras, la actual situación que vive Colombia, los conflictos políticos que hoy se dan en México, la guerra de Irak, cualquier problema serio implica que tú veas sus dimensiones económicas, su entorno geográfico, sus dimensiones sociales, sus efectos políticos, su estructura cultural. Entonces cualquier 
problema serio implica el uso de todas las disciplinas y la idea de Braudel es un poco la de una invitación a la unidisciplinariedad, que también lo plantea Wallerstein. Ellos dicen que tenemos que tratar de hacer un enfoque que rompa efectivamente esta visión de los problemas en disciplinas y vuelva a las visiones holísticas que tuvieron autores como por ejemplo Rousseau, que podía escribir un tratado de economía política y a la vez sobre el tema de la educación, lo mismo un texto de teoría política clásica con elementos propios de la reflexión filosófica. Montesquieu y Hegel hacían lo mismo. Marx no podría entrar en la clasificación nuestra ¿Qué cosa es Marx? ¿Es economista o es filósofo, es jurista o es antropólogo, es geógrafo o es sociólogo? En las categorías de las Ciencias Sociales actuales Marx no queda, porque Marx piensa de otro modo. Es ahí cuando tiene sentido volver a las visiones unidisciplinares de las que te hablaba.

JC: Yapara terminar, ¿̇cuáles son tuspercepciones del ambiente académico en Colombia, qué observas a nivel de comunidades académicas, qué tendencias o quéparadojas identificas en las universidades que has podido visitar en nuestro país, especialmente dentro del campo de las ciencias sociales?

CA: Antes de visitar Colombia -advierto que esta es mi tercera o cuarta visita- yo tenía como una especie de prejuicio. A mediados de los años 90 vine por primera vez a Venezuela a un coloquio sobre Marc Bloch. Yo discutí un poco con los compañeros venezolanos, pero tuve la percepción que era una historiografía y unas ciencias sociales no muy desarrolladas, que les faltaba un poco cierto proceso de maduración, una actualización en los debates contemporáneos, etc. En esa ocasión, dada la continuidad geográfica de Colombia y Venezuela, aproveché para visitar algunos amigos de universidades colombianas de Bogotá y Medellín, y me imaginaba que sería algo parecido, pero por el contrario, quedé muy gratamente sorprendido de los que es la academia colombiana en general. Ustedes están muy actualizados en temas y en autores. Uno va a una librería como por ejemplo la Lerner en Bogotá -aunque los libros son carísimos y esto es una desgracia porque impide un desarrollo intelectual mayor-, y sin embargo encuentras todas las cosas importantes de los debates contemporáneos en historia, en sociología, en ciencia política, en antropología, etc. Han subido mucho el nivel en formación de posgrados. Y cuando discutes con los colegas colombianos observas que el grado de sofisticación del debate es realmente muy alto, incluso pensaría que en un nivel más alto de sofisticación que los debates en México. Paradójicamente uno podría afirmar que quizá por el grado tan grande de complejidad de la propia situación colombiana -porque hay que decirlo, la situación de Colombia es extremamente difícil, extremamente complicada, no es una situación así sencilla de analizar ni sencilla de diagnosticar-, quizá este grado de complejidad de la situación que tiene tantas dimensiones, tantas aristas, tantos matices, produce efectivamente 
científicos sociales que están forzados a complejizar su propio argumento y su formación para entenderla. Eso, digamos entonces, que es bueno. En ese sentido mi diagnóstico es bastante bueno y soy optimista, porque creo que el papel de los intelectuales colombianos puede jugar en ayudar a desentrañar las claves del conflicto actual y a proponer nuevas salidas. $Y$ ya saben que en la línea de "Contrahistorias" siempre tendrán un interlocutor abierto y receptivo de sus propuestas. Muchas gracias.

\section{Pereira - mayo 16 de 2008}

Carlos Antonio Aguirre Rojas (Ciudad de México; 1955), científico social, teórico e investigador mexicano. Doctor en economía por la UNAM.

Su obra es un importante aporte a los textos de divulgación en México, de lo que ha escrito un elevado número de libros, artículos y compilaciones. Es partidario y principal exponente en México de la historia crítica; la tendencia que ha manejado en la mayor parte de sus textos es la de la difusión de las metodologías históricas de mediados del siglo XX, como la escuela de los Anales, asumiendo una postura didáctica.

Sus influencias marcan una línea directa entre los principales exponentes de la historia crítica: Karl Marx, Fernand Braudel, Marc Bloch, Lucien Febvre, Michel Foucault, Carlo Ginzburg, Edward Thompson, Ranajit Guha, Walter Benjamín y Norbert Elías, de los cuales es un importante divulgador en México.

Dado que siempre ha llevado a la praxis su actividad por consecuencia de las posturas ideológicas que representa, es director y fundador de la revista de divulgación científica y cultural Contrahistorias la otra mirada de Clío, fundador del Centro Immanuel Wallerstein en San Cristóbal de las Casas, académico, activo conferencista y adherente a la Sexta Declaración de la Selva Lacandona del EZLN. Basta leer alguno de sus artículos para comprobar su originalidad y estilo ameno y conciso, aunque a veces superficial. Su texto más popular es Antimanual del mal historiador o como hacer una buena historia crítica (Editorial Contrahistorias), en el cual plantea con el tono de un texto de divulgación las discusiones y posturas de la ciencia histórica, en total oposición a la actividad historiográfica en México, que presenta un retraso metodológico respecto al plano internacional. Dicho texto ha tenido una enorme aceptación entre alumnos de nivel medio superior y ha sido reeditado en varios países.

Es doctor en economía por la UNAM y posdoctor en Historia por la École des Hautes Etudes en Sciences Sociales de París. Ha sido profesor invitado en la Universite de Tolouse, en la Universidad Nacional Mayor de San Marcos 
en Lima, Perú. Investigador visitante en el Fernand Braudel Center de la State University of New York y del Centro Juan Marinello en La Habana, Cuba. Actualmente es investigador por la UNAM en el Instituto de Investigaciones Sociales y docente en la Escuela Nacional de Antropología e Historia.

Sus textos han sido traducidos al portugués, inglés, catalán, francés, italiano, alemán, ruso y chino.

\section{FUENTES}

\section{OBRAS DE AGUIRRE ROJAS}

AGUIRRE ROJAS, Carlos Antonio. (1991): Primeras jornadas braudelianas, UNAM. . (1993): Los Anales y la historiografía latinoamericana (UNAM) . (1996): Marc Bloch. Apologíapara la historia o El fficio del historiador FCE. - (2000): Os annales e a bistoriografia francesa: tradicôes críticas de Marc Bloch a Michel Foucault (Universidade Estadual de Maringá). . (2000): Braudel a debate (Imagen Contemporánea) . (2002): Chiapas en perspectiva histórica (El Viejo Topo). . (2003): Braudel, o, mundo e o Brasil, Cortez. . (2003): Antimanual del mal historiador o ¿Cómo hacer una buena historia crítica? Editorial La Vasija, (Actualmente Editorial Contrahistorias, 2005, quinta edición en español). . (2004): Mitos y olvidos en la historia oficial de México, Editorial Era. . (2004): América Latina, Historia e Presente, (Editorial Papirus). . (2004): Fernand Braudel et les sciences humaines, (Editorial L'Harmattan).

(2004): Corrientes, temas y autores de la bistoriografía del siglo XX, (Editorial UJAT). 
(2004): La escuela de los Annales. Ayer, boy mañana, Editorial Era.

. (2004): Die 'Schule’ der Annales, (Leipziger Universitaet Verlag).

. (2004): Immanuel Wallerstein: Critica del sistema mundo capitalista (Estudio y entrevista a Immanuel Wallerstein). Editorial Era.

. (2005): Immanuel Wallerstein . La crisis estructural del capitalismo (Editorial Contrahistorias,).

. (2005): Immanuel Wallerstein . La crisis estructural del capitalismo (Editorial Contrahistorias,). . (2006): Chiapas, planeta tierra, Editorial Contrahistorias.

. (2006): Retratos para la historia. Ensayos de contrabistoria intelectual, (Editorial Contrahistorias.

Contrahistorias).

(2006): América Latina en la encrucijada, (Editorial

CORREA RAMÍREZ, John Jaime. (2008): “Contra la despolitización de la memoria. Entrevista con Carlos Antonio Aguirre Rojas, a propósito de los 40 años de mayo del 68", en Revista Historia de la Educación Latinoamericana No 11, Tunja, Universidad Pedagógica y Tecnológica de Colombia, RUDECOLOMBIA, pp. 77-96 